\title{
Diabetes Mellitus
}

National Cancer Institute

\section{Source}

National Cancer Institute. Diabetes Mellitus. NCI Thesaurus. Code C2985.

A metabolic disorder characterized by abnormally high blood sug ar levels due to diminished production of insulin or insulin resistance/desensitization. 